\title{
Multiple Myeloma: New Perspectives
}

\author{
Ahmed M. Kabel ${ }^{1,2, *}$, Nourah Alqahtani ${ }^{3}$, Rawan Alshehri $^{3}$, Aminah Altalhi $^{3}$, Bashair Alqurashi ${ }^{3}$ \\ ${ }^{1}$ Department of Clinical Pharmacy, College of Pharmacy, Taif University, Taif, KSA \\ ${ }^{2}$ Department of Pharmacology, Faculty of Medicine, Tanta University, Tanta, Egypt \\ ${ }^{3}$ Pharm D Student, College of Pharmacy, Taif University, Taif, KSA \\ *Corresponding author: drakabel@gmail.com
}

\begin{abstract}
Multiple myeloma (MM) is the most common bone malignancy that occurs frequently in older persons. It is the second most common blood cancer in which the patient usually presents with bone pain, nausea, malaise, anemia, renal insufficiency and hypocalcaemia. There is a wide variety of risk factors that may predispose to MM. It is usually discovered on routine laboratory investigations and usually diagnosed with serum or urine protein electrophoresis or immunofixation and bone marrow aspirate analysis. MM often has bad prognosis, although advances in therapy resulted in significant improvement in the overall survival rate. This review sheds light on the incidence, etiology, clinical presentation, the methods of diagnosis, prognosis and the possible lines of management of MM.
\end{abstract}

Keywords: multiple myeloma, risk factors, diagnosis, management, perspectives

Cite This Article: Ahmed M. Kabel, Nourah Alqahtani, Rawan Alshehri, Aminah Altalhi, and Bashair Alqurashi, "Multiple Myeloma: New Perspectives." Journal of Cancer Research and Treatment, vol. 5, no. 3 (2017): 100-104. doi: 10.12691/jcrt-5-3-5.

\section{Introduction}

Multiple myeloma (MM) is one of the malignancies that originate from the plasma cells and usually results in overproduction of light and heavy chain monoclonal antibodies. The specific immunoglobulin produced by the malignant cells is the M-protein which is abundant in serum and/or urine of patients affected with MM [1]. The usual clinical presentation of MM includes bone destruction, hypercalcemia, renal damage, anemia and increased susceptibility to opportunistic infections. The pathogenesis of $\mathrm{MM}$ is poorly understood, although there are increasing data that may link the clinical entity of MM to its predisposing factors. MM often reflects a chromosomal abnormality with many translocations involving chromosomes 13 and 14 [2]. MM affected 488,000 people and resulted in 101,100 deaths in 2015 worldwide [3]. Diagnosis of MM usually depends on detection of abnormal antibodies in blood or urine tests, the presence of malignant plasma cells on bone marrow biopsy and medical imaging finding bone lesions. Also, cases of MM may have a common finding of high blood calcium levels [1]. MM is considered treatable but generally incurable disease. Lines of treatment include systemic corticosteroids, chemotherapy, lenalidomide, thalidomide and stem cell transplantation. Also, bisphosphonates and radiation therapy may be used to reduce pain from bone lesions [4]. This review sheds light on the incidence, etiology, clinical presentation, the methods of diagnosis, prognosis and the possible lines of management of MM.

\section{Risk Factors of MM}

The risk factors for MM are not conclusive, because the cause of MM is yet poorly understood. Most studies suggest that MM has a possible hereditary influence [5]. The risk of MM goes up as people age. Less than $1 \%$ of cases are diagnosed in people younger than 35. Most people diagnosed with $\mathrm{MM}$ are at least 65 years old. Males are slightly more vulnerable to develop MM than females. MM is more common in African Americans than in white Americans with unknown reason [6]. Overweight has been identified as a risk factor for various cancer types. There are two meta-analyses studies that indicated that body mass index (BMI) is positively associated with high risk of MM [7]. Also, farming, occupation as a firefighter, occupation as a hairdresser, exposures to chemicals or pesticides were associated with increased risk of MM. The risk of MM was significantly increased among road and railroad workers, precision metalworkers and workers in transportation and communication industries. Moreover, the risk is increased significantly with certain diseases such as pernicious anemia and ankylosing spondylitis [8].

\section{Pathophysiology of MM}

MM develops in B lymphocytes after they have left the germinal center of the lymph node. The normal cell line most closely associated with MM cells is generally either an activated memory $\mathrm{B}$ cell or the precursor to plasma cells, the plasmablasts [3]. The immune system keeps the 
proliferation of $\mathrm{B}$ cells and the secretion of antibodies under tight control. When chromosomes and genes are damaged, this control is lost. Often, a promoter gene moves to a certain chromosome where it stimulates an antibody gene to overproduction [1].

A chromosomal translocation between the immunoglobulin heavy chain gene on chromosome 14, locus q32 and an oncogene (often 11q13, 4p16.3, 6p21, 16q23 and 20q11) is frequently observed in patients with MM [7]. This mutation results in dysregulation of the oncogene which is thought to be an important initiating event in the pathogenesis of MM. The result is proliferation of a plasma cell clone and genomic instability that leads to further mutations and translocations. The chromosome 14 abnormality is observed in about $50 \%$ of all cases of myeloma. Deletion of parts of chromosome 13 is also observed in about $50 \%$ of cases [9]. Production of cytokines by the plasma cells causes much of their localized damage, such as osteoporosis and creates a microenvironment in which malignant cells can survive. The produced antibodies are deposited in various organs, leading to polyneuropathy, renal failure and various other symptoms [10].

\section{Classification of MM}

MM is not one disease but includes many types and subtypes. Each subtype is largely defined by the specific genetic and cytogenetic mutation. Classification of MM can be proposed at three levels including biological genetic, prognostic and predictive classification [11].

\subsection{Biological Genetic Classification}

This classification mostly depends on the biology-based considerations. The groups created by this classification have unique clinical and prognostic implications, but this is not a prerequisite. Examples of this classification include the hyperdiploid versus non-hyperdiploid classification and the specific chromosomal translocations. The subgroups in this classification will be defined primordially by the primary genetic abnormality that will be associated with a constellation of other genetic changes associated with clone progression and evolution [12].

\subsection{Prognostic Classification}

Prognostic classification helps the physicians to discriminate the outcome of patients treated with multiple therapeutic modalities. Factors capable of serving as prognostic determinants will be associated with baseline clinicopathological features of disease aggressiveness such as high proliferation rates, high $\beta_{2}$-microglobulin, hypercalcemia, extramedullary disease and elevated lactate dehydrogenase [13].

\subsection{Predictive Classification}

Most currently available therapies for MM are not thought to be targeted therapies and thus used in all suitable candidates. Yet only a fraction of cases exhibit response to single agent therapy, suggesting that subgroups of MM may be more or less responsive to specific therapies. Assays were developed to predict patients who are likely to benefit from bortezomib early in the course of therapy and are able to identify patterns associated with disease responsiveness after drug administration [14].

\section{Clinical Presentation of MM}

The presenting signs and symptoms of MM include anemia, weakness, bone pain, pathologic fractures, pneumococcal infection, hypercalcemia, renal failure or spinal cord compression. The most common presenting symptoms are fatigue and bone pain. Osteolytic bone lesions and compression fractures are the hallmark of the disease [1]. Anemia occurs in $70 \%$ of patients at diagnosis and is the primary cause of fatigue. Renal dysfunction occurs in $50 \%$ and hypercalcemia in $25 \%$ of patients. In some patients, large amounts of myeloma protein can increase the blood viscosity which may slow the blood flow to the brain and cause confusion, dizziness and symptoms of cerebrovascular stroke such as weakness on one side of the body and slurred speech. Sometimes, MM may be asymptomatic and may not have laboratory test results that require treatment. This type of $\mathrm{MM}$ is called smoldering myeloma [2].

\section{Complications of MM}

Renal failure is a common complication of MM that may occur in $20-40 \%$ of newly diagnosed patients and may help diagnosis and represent a major obstacle to management [15]. Anemia, bone marrow failure and bleeding disorders may occur in about $75 \%$ of patients with $\mathrm{MM}$ at diagnosis [16]. Also, bone complications including pathologic fractures, spinal cord compression and hyercalcemia may occur in about $80-90 \%$ of patients with MM during the course of the disease. These lesions are mainly osteolytic and are associated with severe bone pain, pathologic fractures, as well as increased mortality. Multiple myeloma bone disease is distinct from the bone disease caused by other types of tumors that metastasize to bones and is characterized by dysfunction of both bone formation and bone resorption [17].

Patients with MM may have neurologic complications including peripheral neuropathies, cranial nerve palsies, spinal radiculopathies, spinal cord compression and metabolic encephalopathies. The precipitating factors of these complications include vertebral compression fractures, base of skull and other bony involvement, metabolic disorders, hyperviscosity syndrome and amyloidosis. Involvement of the central nervous system in $\mathrm{MM}$ is rare, either as presumed intracerebral metastases or as leptomeningeal myelomatosis [18]. Also, MM is associated with an increased incidence of bacterial and viral infections due to defects in cellular and humoral immunity, reduced mobility and performance status, which are all associated with both the disease and its treatment. It was reported that even up to $10 \%$ of patients with $\mathrm{MM}$ die due to secondary infections within two months of diagnosis [19]. 


\section{Diagnosis of MM}

Diagnosis of MM is somewhat difficult because it's an uncommon type of malignancy that initially has no symptoms in most patients. Urine and blood tests are needed to check for certain types of antibodies and proteins. If $\mathrm{MM}$ is suspected, the hematologist will need further tests and scans to confirm the diagnosis and determine the suitable lines of management [20]. There are various blood tests that are used to diagnose MM that may need to be repeated to monitor the condition. They include erythrocyte sedimentation rate (ESR), plasma viscosity (PV), complete blood count (CBC), blood calcium level, liver and kidney function tests. Urine tests may show $\mathrm{M}$ proteins, which are called Bence Jones proteins when they're detected in urine [21].

$\mathrm{X}$-rays and other scans possess a key role in staging of newly diagnosed and relapsed MM. Magnetic resonance imaging gives information complementary to skeletal survey and is recommended in MM patients with normal conventional radiography and in patients with an apparently solitary plasmacytoma of bone [22]. Bone marrow biopsy and sampling of bone may be needed to check for cancerous plasma cells and to confirm MM [23].

\section{Treatment of MM}

There are several types of non-chemotherapy drugs that have emerged in the past decade as important options for the treatment of MM [24]. These drugs may be options both for newly diagnosed patients and at the time of relapse. In most cases, these drugs are used in combination with dexamethasone, with each other, or with standard chemotherapeutic agents [25]. In addition to direct treatment of the plasma cell proliferation, bisphosphonates are used to prevent pathological fractures. Also, bisphosphonates were observed to have direct anti-tumor effect even in patients without known skeletal disease. Red blood cell transfusions or erythropoietin may be used for management of severe anemia. Bortezomib is the first therapeutic proteosome inhibitor approved by the U.S. FDA for treatment of relapsed MM [26].

\subsection{Initial Therapy}

The initial treatment of MM depends mainly on the age of the patient and the associated comorbidities. Recently, high-dose chemotherapy with autologous hematopoietic stem-cell transplantation has become the main line of treatment for patients under the age of 65 years [27]. Prior to stem-cell transplantation, these patients receive an initial course of induction chemotherapy. The most commonly used induction regimens are bortezomib based regimens and dexamethasone-lenalidomide [28]. The term "Autologous stem cell transplantation" refers to transplantation of a patient's own stem cells after chemotherapy. It is not curative, but prolongs the overall survival rate and produces complete remission [29]. Allogeneic stem cell transplantation means transplantation of a healthy person's stem cells into the affected patient which may have the potential for cure, but is used in a very low percentage of patients. Also, this line of treatment may have a 5-10\% treatment-associated mortality rate which makes it not a constituent of the initial therapy [30].

For patients over the age of 65 years who often cannot tolerate stem cell transplantation, the preferred line of treatment has been chemotherapy with melphalan and prednisone. Recent studies suggest improved outcomes in patients over the age of 65 years who cannot tolerate stem cell transplantation with new chemotherapy regimens such as with bortezomib and lenalidomide [31]. However, lenalidomide may cause significant myelosuppression. Also, chemotherapy-induced peripheral neuropathy and thrombocytopenia are frequently encountered adverse effects with bortezomib [32].

\subsection{Maintenance Therapy}

After completion of the initial treatment, an ongoing maintenance therapy is often needed to prevent relapse and improve the survival rate. In young patients, maintenance therapy with thalidomide appears to decrease the tumor burden which is reflected as prolonged progression-free survival. In addition to thalidomide, lenalidomide, or bortezomib may be used to improve the therapeutic outcomes in these cases [33].

\subsection{Management of Relapse}

In the majority of cases of MM, relapse frequently occurs following treatment which may be due to tumor heterogeneity. Management of relapsed cases usually depends on the patient's condition, the previous treatment modalities used and the duration of remission [34]. Lines of treatment of relapsed disease include re-treatment with the original agent, the use of other drugs such as cyclophosphamide, melphalan, thalidomide or dexamethasone, and introduction of a second autologous stem cell transplant [35].

Resistance to treatment may occur later in the course of the disease which is usually reversible. Some new treatment modalities may re-sensitize the tumor to standard lines of therapy. For patients with relapsed disease, bortezomib is the preferred line of treatment. Also, lenalidomide and pomalidomide, which are less toxic thalidomide analogues, are showing a promising effect for treatment of relapsed and refractory cases of MM [34]. Ixazomib is an orally available proteasome inhibitor indicated in combination with lenalidomide and dexamethasone in patients who had received at least one prior therapy. Panobinostat is a histone deacetylase inhibitor used in combination with bortezomib and dexamethasone in patients who had received at least 2 prior chemotherapy regimens, including bortezomib and an immunomodulatory agent such as lenalidomide or pomalidomide [36]. Carfilzomib is a proteasome inhibitor that is indicated either as a single agent for therapy of patients with relapsed or refractory MM who had received one or more lines of therapy or in combination with dexamethasone or with lenalidomide + dexamethasone for treatment of patients with relapsed or refractory MM who had received one to three lines of therapy [37]. Daratumumab is a monoclonal antibody that is directed against CD38 indicated for treatment of patients with MM who had received at least three prior 
lines of therapy including a proteasome inhibitor and an immunomodulatory agent or who are double refractory to an immunomodulatory agent and a proteasome inhibitor. All of these drugs seem to maintain the monoclonal peak at a reasonable level and raise the life expectancy of cases of relapse of MM [28].

\subsection{Palliative Care}

Recent studies recommended early palliative care for patients with advanced $\mathrm{MM}$ at the time of diagnosis as well as for anyone who has significant symptoms. Palliative care can be introduced at any stage of the tumor and can be provided at the same time with the curative treatment. In addition to reducing the symptoms of cancer, palliative care helps to manage the unwanted adverse effects, such as pain, nausea and vomiting related to chemotherapy [38].

\subsection{The Role of Immunotherapy in Management of MM}

Standard treatments for MM include standard and highdose chemotherapy and proteosome inhibitors that are usually given in combination with corticosteroids in the absence or presence of stem cell transplantation. However, MM remains incurable and relapse is inevitable in the majority of cases [3]. Immunotherapy was developed to be the only line of treatment that may be associated with long-term complete remission in patients with MM. Immunotherapies for MM usually act on three main approaches (Figure 1). The first one is to reverse tumor mediated immune paralysis which can be achieved either by immunomodulatory drugs, immune checkpoint inhibitors or cytokines such as interferons, granulocyte macrophage colony stimulating factors and siltuximab [39]. The second approach is to stimulate myeloma specific immune responses either by using MM vaccines or adoptive T-cell Transfer (e.g., chimeric antigen receptor T-cells) [40]. The third approach is to selectively eliminate the malignant clones by monoclonal antibodies such as daratumumab and elotuzumab [41].

\section{Prognosis of MM}

Multiple myeloma is highly treatable but rarely curable disease. The median survival rate in the prechemotherapy era was about 7 months. MM is potentially curable when it presents as a solitary plasmacytoma of bone or as an extramedullary plasmacytoma. The prognosis was significantly improved in the last years due to the emergence of sensitive laboratory tests that help early diagnosis and thereby early introduction of different therapeutic agents [2]. The prognostic indicators help to determine the rate of growth of the tumor, the extent of metastasis, the biologic make-up of the tumor, the response to therapy, and the overall survival rate of the patients. The prognosis of MM often depends on the stage of the disease, whether a certain immunoglobulin is present, the extent of involvement of the kidney, the response of the tumor to the initial treatment, the recurrence of the tumor, the age and general health of the patient [42]. Also, the prognosis is variable according to the type of treatment used. For example, the overall survival rate with conventional therapy is about 3 years, and event-free survival is less than 2 years. On the other hand, the survival rate with high-dose chemotherapy with stem-cell transplantation is greater than $50 \%$ at 5 years [43].

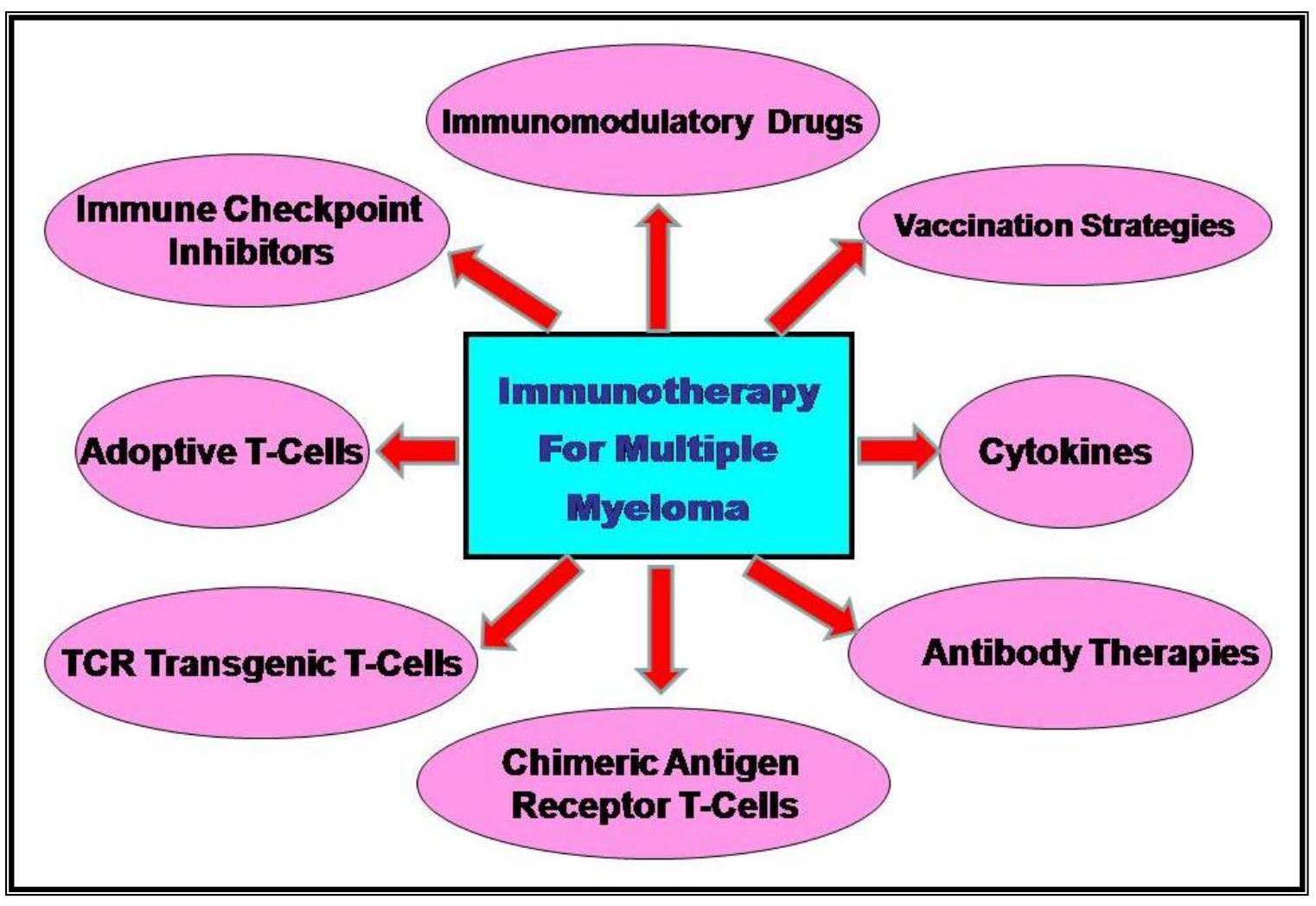

Figure 1. Immunotherapy for Multiple Myeloma 


\section{Conclusion}

MM is not one disease but rather many, with each one of the subtypes largely defined by the specific genetic and cytogenetic aberrations. The risk factors for multiple myeloma are poorly understood .Researchers believe that multiple myeloma is most likely the result of many risk factors acting together. Multiple myeloma symptoms may vary by patient, with the early stages of myeloma often presenting no visible symptoms or signs. There are several types of non-chemotherapy drugs that have emerged in the past decade as important options for the treatment of multiple myeloma. The main types of treatment for multiple myeloma are drug therapy, surgery, stem cell transplant, radiation and chemotherapy. The multiple myeloma is highly treatable but rarely curable. The median survival in the prechemotherapy era was about 7 months and are many Specific factors affecting a multiple myeloma prognosis.

\section{References}

[1] Al-Farsi K. Multiple Myeloma: An Update. Oman Medical Journal. 2013; 28(1): 3-11.

[2] Laubach J, Richardson P, Anderson K. Multiple myeloma. Annu Rev Med. 2011; 62: 249-64.

[3] Kumar SK, Callander NS, Alsina M, Atanackovic D, Biermann JS, Chandler JC, et al. Multiple Myeloma, Version 3. 2017, NCCN Clinical Practice Guidelines in Oncology. J Natl Compr Canc Netw. 2017; 15(2): 230-69.

[4] Pingali SR, Haddad RY, Saad A. Current concepts of clinical management of multiple myeloma. Dis Mon. 2012; 58(4): 195207.

[5] Koura DT, Langston AA. Inherited predisposition to multiple myeloma. Therapeutic Advances in Hematology. 2013; 4(4): 291-7.

[6] Sergentanis TN, Zagouri F, Tsilimidos G, Tsagianni A, Tseliou M, Dimopoulos MA, et al. Risk Factors for Multiple Myeloma: A Systematic Review of Meta-Analyses. Clin Lymphoma Myeloma Leuk. 2015; 15(10): 563-77.

[7] Wallin A, Larsson SC. Body mass index and risk of multiple myeloma: a meta-analysis of prospective studies. Eur J Cancer. 2011; 47(11): 1606-15.

[8] Rajkumar SV. Multiple myeloma: 2016 update on diagnosis, risk-stratification, and management. Am J Hematol. 2016; 91(7): 719-34.

[9] Kyle RA, Rajkumar SV. Multiple myeloma. N. Engl. J. Med. 2004; 351 (18): 1860-73.

[10] Tricot G. New insights into role of microenvironment in multiple myeloma. Lancet. 2000; 355 (9200): 248-50.

[11] Fonseca R, Monge J. Myeloma: classification and risk assessment. Semin Oncol. 2013; 40(5): 554-66.

[12] Fonseca R, Monge J, Dimopoulos MA. Staging and prognostication of multiple myeloma. Expert review of hematology. 2014; 7(1): 21-31.

[13] Kyle R, Rajkumar S. Criteria for diagnosis, staging, risk stratification and response assessment of multiple myeloma. Leukemia: official journal of the Leukemia Society of America, Leukemia Research Fund, UK. 2009; 23(1):3-9.

[14] Bergsagel PL, Chesi M. V. Molecular classification and risk stratification of myeloma. Hematol oncol. 2013; 31(0 1): 38-41.

[15] Terpos E, Kleber M, Engelhardt M, et al. European Myeloma Network Guidelines for the Management of Multiple Myeloma-related Complications. Haematologica. 2015; 100(10): 1254-66

[16] Gay F, Palumbo A. Management of disease- and treatment-related complications in patients with multiple myeloma. Med Oncol. 2010; 27 Suppl 1: S43-52.
[17] Hameed A, Brady JJ, Dowling P, Clynes M, O’Gorman P. Bone Disease in Multiple Myeloma: Pathophysiology and Management. Cancer Growth and Metastasis. 2014; 7: 33-42.

[18] Sullivan E, Smith LC, Falco AM. Multiple Myeloma: Cast Nephropathy, VTE, and Neurologic Complications. J Adv Pract Oncol. 2013; 4(1): 37-46.

[19] Gay F, Palumbo A. Multiple myeloma: management of adverse events. Med Oncol. 2010; 27(3): 646-53.

[20] Eslick R, Talaulikar D. Multiple myeloma: from diagnosis to treatment. Aust Fam Physician. 2013; 42(10): 684-8.

[21] Michels TC, Petersen KE. Multiple Myeloma: Diagnosis and Treatment. Am Fam Physician. 2017; 95(6): 373-83.

[22] Gerecke C, Fuhrmann S, Strifler S, Schmidt-Hieber M, Einsele H, Knop S. The Diagnosis and Treatment of Multiple Myeloma. Dtsch Arztebl Int. 2016; 113(27-28): 470-6.

[23] Rajkumar SV, Kumar S. Multiple Myeloma: Diagnosis and Treatment. Mayo Clin Proc. 2016; 91(1):101-19.

[24] Kabel AM, Abd Elmaaboud MA. Cancer: Role of Nutrition, Pathogenesis, Diagnosis and Management. World Journal of Nutrition and Health 2014; 2(4): 48-51.

[25] Munshi NC, Anderson KC. New strategies in the treatment of multiple myeloma. Clin Cancer Res. 2013; 19(13): 3337-44.

[26] Mina R, Cerrato C, Bernardini A, Aghemo E, Palumbo A. New pharmacotherapy options for multiple myeloma. Expert Opin Pharmacother. 2016; 17(2): 181-92.

[27] Jasielec JK, Jakubowiak AJ. Current approaches to the initial treatment of symptomatic multiple myeloma. Int J Hematol Oncol. 2013; 2(1): 10.2217/ijh.13.3.

[28] El-Amm J, Tabbara IA. Emerging therapies in multiple myeloma. Am J Clin Oncol. 2015; 38(3): 315-21.

[29] Mohty M, Harousseau J-L. Treatment of autologous stem cell transplant-eligible multiple myeloma patients: ten questions and answers. Haematologica. 2014; 99(3): 408-16.

[30] Bensinger W, Rotta M, Storer B, et al. Allogeneic Stem Cell Transplantation for Multiple Myeloma: A Review of Outcomes at a single Transplant Center. Bone marrow transplant. 2012; 47(10): 1312-7.

[31] Torimoto Y, Shindo M, Ikuta K, Kohgo Y. Current therapeutic strategies for multiple myeloma. Int J Clin Oncol. 2015; 20(3): 423-30.

[32] Zhang X, Chen W-W, Huang W-J. Chemotherapy-induced peripheral neuropathy. Biomed Rep. 2017; 6(3): 267-71.

[33] Mewawalla P, Chilkulwar A. Maintenance therapy in multiple myeloma. Ther Adv Hematol. 2017; 8(2): 71-9.

[34] Sonneveld P, Broijl A. Treatment of relapsed and refractory multiple myeloma. Haematologica. 2016; 101(4): 396-406.

[35] Castelli R, Gualtierotti R, Orofino N, Losurdo A, Gandolfi S, Cugno M. Current and Emerging Treatment Options for Patients with Relapsed Myeloma. Clin Med Insights Oncol. 2013; 7: 209-19.

[36] Cornell RF, Kassim AA. Evolving paradigms in the treatment of relapsed/refractory multiple myeloma: increased options and increased complexity. Bone Marrow Transplant. 2016; 51(4): 479-91.

[37] Raza S, Safyan RA, Rosenbaum E, Bowman AS, Lentzsch S Optimizing current and emerging therapies in multiple myeloma: a guide for the hematologist. Ther Adv Hematol. 2017; 8(2): 55-70.

[38] Cömert M, Güneş AE, Şahin F, Saydam G. Quality of Life and Supportive Care in Multiple Myeloma. Turk J Haematol. 2013; 30(3): 234-46.

[39] Kocoglu M, Badros A. The Role of Immunotherapy in Multiple Myeloma. Emadi A, Desai D, eds. Pharmaceuticals. 2016; 9(1): 3.

[40] Neri P, Bahlis NJ, Lonial S. New Strategies in Multiple Myeloma: Immunotherapy as a Novel Approach to Treat Patients with Multiple Myeloma. Clin Cancer Res. 2016; 22(24): 5959-65.

[41] Chung C. Role of Immunotherapy in Targeting the Bone Marrow Microenvironment in Multiple Myeloma: An Evolving Therapeutic Strategy. Pharmacotherapy. 2017; 37(1): 129-43.

[42] Hanbali A, Hassanein M, Rasheed W, Aljurf M, Alsharif F. The Evolution of Prognostic Factors in Multiple Myeloma. Advances in Hematology 2017; 2017: 4812637.

[43] Gupta A, Kumar L. Evolving role of high dose stem cell therapy in multiple myeloma. Indian J Med Paediatr Oncol. 2011; 32(1): $17-24$. 